\title{
Imagem corporal de pessoas com úlceras venosas
}

\section{Body image of people with venous ulcers}

\section{Geraldo Magela Salomé}

Universidade do Vale do Sapucaí (Pouso Alegre). Minas Gerais, Brasil. salomereiki@univas.edu.br

RESUMO | OBJETIVO: avaliar a imagem corporal em pessoas com úlcera venosa. MÉTODOS: estudo descritivo realizado em um Ambulatório de Feridas, localizado na cidade de Pouso Alegre, Minas Gerais. Participaram do estudo 30 pessoas com úlceras venosas. Dois instrumentos foram utilizados: um questionário para a coleta de dados sociodemográficos e clínicos e a escala Body Investment Scale (BIS). RESULTADOS: entre os 30 participantes, $16(53,3 \%)$ eram do sexo feminino e $14(46,7 \%)$, masculino; 25 (83,3\%) tinham idade acima de 60 anos e 20 $(66,7 \%)$ eram casados. A média do escore total na escala BIS foi de 34,0; e as médias dos escores para os domínios imagem corporal, cuidado pessoal, proteção corporal e toque corporal foram 16,30, 23,93, 5,53 e 18,30. Tais achados caracterizam que esses indivíduos com úlcera venosa que participaram do estudo apresentaram sentimentos negativos relacionados à própria autoestima, autoimagem e à piora na qualidade de vida. CONCLUSÃO: os resultados do estudo apontaram que os indivíduos com úlceras venosas apresentavam alterações nos domínios imagem corporal, proteção corporal e no toque corporal, ou seja, manifestaram ter sentimentos negativos com relação ao seu corpo.

DESCRITORES:Autoimagem. Imagem Corporal.Úlceravaricosa. Úlcera da perna.

\begin{abstract}
OBJECTIVE: to assess body image in people with venous ulcers. METHODS: descriptive study conducted at the Wound Ambulatory located in the city of Pouso Alegre, Minas Gerais. Thirty people with venous ulcers participated in the study. Two instruments were used: a questionnaire for collecting sociodemographic and clinical data and the Body Investment Scale (BIS). RESULTS: among the 30 participants, $16(53.3 \%)$ were female and $14(46.7 \%)$ male, 25 (83.3\%) were over 60 years old and 20 (66.7\%) were married. The average of the total score on the BIS scale was 34.0; and the mean scores for the body image, personal care, body protection and body touch domains were 16.30, 23.93, 5.53 and 18.30. Such findings characterizing that these individuals have a negative image. Patients with venous ulcers showed changes in the body image, body protection and body touch domains. CONCLUSION: the results of the study showed that individuals with venous ulcers had alterations in the domains of body image, body protection and body touch, that is, they manifested negative feelings about their body.
\end{abstract}

DESCRITORES: Self image. Body image. Varicose ulcer. Leg ulcer. 


\section{Introdução}

A úlcera de perna é uma doença crônica que pode ser desencadeada a partir de transtornos hematológicos, vasculares ou metabólicos. A insuficiência valvular crônica é a principal causadora das ulcerações, respondendo por quase $80 \%$ de todas as úlceras venosas que afetam aproximadamente $1 \%$ da população mundial, causando aumento nas despesas de saúde, alteração na qualidade de vida, na autoimagem, na imagem corporal, no sono e na capacidade funcional ${ }^{1-2}$.

A instalação da úlcera venosa geralmente é lenta; os pacientes podem referir um trauma prévio de membro inferior, varizes e até mesmo trombose venosa profunda. O edema é frequente, além da sensação de dor que se agrava no final da tarde devido à permanência na posição em pé ou sentado durante o dia. Os profissionais de saúde orientam o paciente para que o membro seja elevado ao final do dia, com a finalidade de diminuir a dor e o edema. Pode ocorrer eczema ao redor da ulceração, com eritema, prurido e exsudato. Após o diagnóstico clínico, são necessários exames para a verificação das alterações anatômicas venosas que levaram à formação da úlcera; podendo-se identificar se ela foi provocada por uma obstrução ou por refluxo venoso ${ }^{3}$.

Depois de feito o diagnóstico, a atenção deve ser voltada para o tratamento da úlcera venosa e para evitar as recidivas. Os tratamentos mais utilizados são: terapia compressiva como uso da bota de Unna ou bandagem elástica, uso de coberturas que promovem o processo de cicatrização e a intervenção cirúrgica na anormalidade venosa $a^{1,4-5}$.

Em razão da demora do processo da cicatrização da úlcera venosa, a pessoa passa por desconforto físico e psicológico, o que limita as suas atividades diárias. A presença de dor e de odor causado pelo exsudado faz com que a mesma se isole, sinta-se deprimida, com baixa autoestima, tenha distorção da autoimagem e piora na qualidade de vida, podendo levar o paciente a desistir do tratamento ${ }^{6-7}$.
Estudos realizados com pessoas com úlcera venosa, nos quais foi avaliado o impacto dessa lesão em suas vidas, os autores concluíram que, ao serem acometidas por úlcera venosa, elas apresentam dor, exsudado e edemas de membros, causando alterações na autoestima, autoimagem e na qualidade de vida e sensação de impotência ${ }^{8-9}$. Em outros estudos, os autores concluíram que após o indivíduo contrair a ferida, ele poderá apresentar várias alterações na vida diária: dor, baixa autoestima e isolamento social. Entretanto os autores assinalaram que as pessoas que tiveram a úlcera cicatrizada apresentaram redução da intensidade da dor, melhora da autoestima e da qualidade de vida ${ }^{10-11}$.

A autoimagem ou imagem corporal reflete como o indivíduo se vê com relação ao seu aspecto físico. Em contraste, a autoestima refere-se ao sentimento do indivíduo com relação à sua autoimagem. Portanto, a autoimagem tem influência direta na autoestima. Assim, uma pessoa com baixa autoimagem terá também uma baixa autoestima ${ }^{10-13}$. Cabe à equipe de saúde prestar assistência sistematizada e individualizada, por meio da melhor conduta, com a escolha da cobertura ideal que promova a cicatrização da lesão, encaminhando o paciente para avaliação do psicólogo. Tal conduta tem como meta melhorar e amenizar o sofrimento psicoemocional e promover a socialização dos indivíduos com úlcera venosa.

Durante a prestação dos cuidados aos pacientes com úlcera venosa, os profissionais devem estar atentos às mudanças de comportamentos. A abordagem inicial é fundamental para o direcionamento ao serviço de saúde especializado, que possibilitará o diagnóstico precoce, bem como o prognóstico para esses indivíduos. É necessário que se tenha conhecimento do grau de insatisfação com a imagem corporal por parte das pessoas com úlcera venosa de diferentes contextos, já que a percepção da imagem corporal pode ser influente na aceitação e continuidade do tratamento e na cicatrização de úlcera. O resultado desta pesquisa pode subsidiar os profissionais que atuam com o público a trabaIharem a temática "corpo nas pessoas com ferida".

Diante disso, o estudo aqui descrito teve como objetivo avaliar a imagem corporal em pessoas com úlcera venosa. 


\section{Métodos}

Estudo descritivo e quantitativo desenvolvido no Ambulatório de Feridas (NAEEnf), localizado na cidade de Pouso Alegre-MG. Os dados foram coletados no período compreendido entre março a maio de 2018, após aprovação pelo Comitê de Ética em Pesquisa da Faculdade de Ciências da Saúde Dr. José Antônio Garcia Coutinho da Universidade do Vale do Sapucaí (UNIVÁS), Pouso Alegre-MG, (parecer número 276.259).

Os dados foram coletados pelo próprio pesquisador em uma sala previamente preparada, garantindo o conforto e a tranquilidade do paciente para a emissão das respostas.

Após levantamento do número das pessoas com úlcera venosa atendidas no ambulatório, foram identificados 42 pacientes com essa lesão. A amostra foi selecionada de forma não probabilística, por conveniência e constituída de trinta indivíduos com úlcera venosa, que atenderam os seguintes critérios de inclusão: indivíduos com idade acima de 18 anos e índice tornozelo/braço entre 0,9 e 1,4. Pacientes com úlcera mista ou arterial e pacientes diabéticos com úlcera no pé não foram incluídos na amostra.

Dois instrumentos foram utilizados para a coleta de dados. Em relação ao perfil sociodemográfico, foi elaborado um instrumento com as seguintes variáveis: sexo, faixa etária e estado civil. Para avaliar a imagem corporal, foi adotado o instrumento Body Investment Scale (BIS).
A versão brasileira do BIS ou Questionário de Investimento Corporal é composta por vinte itens distribuídos em três domínios (imagem corporal, cuidado corporal e toque corporal).14 As opções de respostas são apresentadas em uma escala Likert de cinco pontos, indo de "discordo totalmente" a "concordo totalmente". O escore final da escala é obtido pela reversão dos escores dos itens $2,5,9,11,13$ e 17, e soma dos escores de todos os itens. O escore total varia entre 20 e 100. Quanto maior o valor do escore total, maior será o sentimento positivo em relação à imagem corporal. No estudo de adaptação transcultural da versão brasileira da escala BIS,14 foi feita uma análise fatorial exploratória com rotação varimax. $\mathrm{Na}$ versão brasileira da escala, foram mantidos 20 itens dos 24 itens originais, com 4 fatores explicando 36,3\% da variância total da escala. Imagem corporal (fator 1 ) apresentou Cronbach $a=0,81$; cuidado corporal (fator 2 ) obteve $a$ = 0,70; toque corporal (fator 3 ) apresentou $a=0,66$; e proteção corporal (fator 4) obteve $\alpha=0,371^{14}$.

Os dados foram compilados em uma planilha do programa Microsoft ${ }^{\circledR}$ Office 365, versão 1812, e, após codificação e tabulação, foram analisados por meio de estatística descritiva. Foi realizado o teste QuiQuadrado para independência e considerando nível de significância de $5 \%(p \leq 0,05)$.

\section{Resultados}

Na Tabela 1, observou-se que a maioria dos pacientes que participaram do estudo era do sexo feminino ( $n=16 ; 53,3 \%$ ) e a média de idade foi 64 anos.

Tabela 1. Características sociodemográficas dos participantes do estudo Pouso Alegre, Minas Gerais, 2018

\begin{tabular}{lcc}
\hline Variáveis & N & $\%$ \\
\hline Sexo & 14 & \\
Masculino & 16 & 46,7 \\
Feminino & & 53,3 \\
& & \\
Faixas de idade & 1 & 3,3 \\
35 a 45 anos & 4 & 13,3 \\
46 a 55 anos & 25 & 83,3 \\
Acima de 60 anos & & \\
& 6 & 20,0 \\
Estado civil & 20 & 66,7 \\
Separado & 4 & 13,3 \\
Casado & $\mathbf{3 0}$ & $\mathbf{1 0 0}$ \\
\hline Outros & & \\
\hline Total & & \\
\hline
\end{tabular}


A Tabela 2 mostra que a média do escore total da escala BIS foi baixa na população estudada. Segundo esse resultado, os pacientes do estudo apresentam baixa imagem corporal. Houve diferença estatística entre os valores da média e mediana do escore total na escala BIS. A média do escore total e dos domínios "imagem corporal, toque corporal e "Proteção corporal" da escala BIS foi baixa na população estudada. Houve diferença significativa entre as médias dos domínios da escala BIS. De acordo com esse achado, os pacientes do estudo apresentam baixa imagem corporal.

Tabela 2. Domínios da escala de Body Investment Scale em pacientes com úlcera venosa. Pouso Alegre, Minas Gerais, 2018

\begin{tabular}{lccccc}
\hline & \multicolumn{4}{c}{ Domínio } \\
\cline { 2 - 5 } Estatística Descritiva & Imagem corporal & Cuidado pessoal & Toque corporal & $\begin{array}{c}\text { Proteção } \\
\text { corporal }\end{array}$ & Valor do $\mathbf{p}$ \\
\hline Média & 16,30 & 23,93 & 18,30 & 5,53 & $0,003^{*}$ \\
Desvio-Padrão & 2,806 & 3,248 & 1,985 & 1,592 & \\
Mediana & 16,00 & 23,00 & 19,00 & 6,00 \\
& & & & & \\
\hline
\end{tabular}

*Nível de significância $p \leq 0,05$

\section{Discussão}

No estudo em questão, verificou-se predomínio de sexo feminino e de indivíduos com idade acima de 60 anos. Esses resultados são similares aos resultados de outros estudos que revelam uma tendência maior da ocorrência de úlceras venosas entre pessoas do sexo feminino e idosos ${ }^{6,14}$. A média do escore total na escala BIS foi 34,0. Os resultados ora encontrados permitem confirmar que os pacientes que participaram do estudo apresentavam baixa autoimagem. Vários autores corroboram esses achados, ${ }^{6,14-16}$. A autoimagem é considerada um importante indicador de saúde mental por interferir em aspectos afetivos, sociais e psicológicos.

A úlcera venosa pode apresentar exsudato, odor, edema, dor e dificuldade na cicatrização. Tais fatores podem resultar em imagem distorcida e diminuição da autoimagem e da autoestima, repercutindo na percepção sobre si mesmo, ou seja, a pessoa se vê sem atrativos. A mudança súbita da imagem corporal ocasiona confusão e alteração negativa na forma como ela se percebe ${ }^{17-19}$. O conhecimento dos níveis de autoimagem relacionados à saúde em pacientes com úlcera venosa, bem como sobre as mudanças provocadas no seu cotidiano e nas atividades diárias, fornece ao profissional subsídio para o planejamento da assistência a esses indivíduos. Assim, os profissionais da área de saúde poderão desenvolver programas preventivos e estratégias de intervenção que minimizem os transtornos decorrentes de alterações físicas e anatômicas que acontecem com a pessoa com úlcera venosa.

Os baixos escores nos domínios da escala BIS observados no presente estudo coincidem com os resultados de trabalhos prévios que indicam que a úlcera venosa afeta negativamente vária dimensões relacionadas à autoimagem, destacando-se os domínios proteção corporal, toque corporal e imagem corporal que se mostraram mais afetados. O paciente com úlcera venosa defronta-se com uma multiplicidade de alterações de ordem física, biológica, psicológica, espiritual, social e sexual, as quais geram impacto sobre a imagem corporal, autoestima e qualidade de vida. Tal impacto influencia nas atividades diárias do paciente, como viajar, praticar esporte, frequentar clubes, bem como interfere no autocuidado devido à insegurança, ao medo em relação à aderência do curativo, ao odor exalado pela úlcera e às frequentes trocas do curativo. Essas alterações podem ocasionar afastamento do trabalho no indivíduo acometido pela referida lesão 20,21 . 
A presença da úlcera gera a necessidade da prática do autocuidado pelo paciente, visando recuperar a sua saúde e restabelecer suas atividades da vida diária. Porém a úlcera venosa é uma doença crônica de tratamento complexo, o que faz com que o paciente encontre dificuldades para executar o autocuidado. O paciente precisa participar ativamente no seu autocuidado para que o tratamento seja bem-sucedido. Muitas vezes isso requer mudanças de estilo de vida adotando-se práticas mais saudáveis. Um resultado positivo não depende somente do tratamento prescrito pelo profissional de saúde. A pessoa com úlcera venosa precisa ser conscientizada sobre sua condição de saúde e sobre a necessidade de práticas voltadas para a promoção da saúde e qualidade de vida e da importância de sua adesão ao tratamento ${ }^{6,18}$.

Na maioria das vezes, as pessoas com úlceras venosas mobilizam saberes de forma independente ao realizar o próprio cuidado, sempre de acordo com o que consideram ser o melhor para si. Ou seja, para cuidar de si, elas lançam mão do conhecimento sedimentado a partir da própria experiência com a doença e do cuidado por familiares que experienciaram a mesma condição ${ }^{19,22}$. Quando isso não é possível, solicitam aos prestadores de cuidados formais e informais que as orientem nas ações de cuidado. Contudo é importante ter em mente que as pessoas seguem os conselhos dos profissionais de saúde quando os compreendem e quando acreditam que lhes trazem benefícios atingíveis. Daí a importância da adequação da informação à condição das pessoas, a fim de que o ensinamento seja assimilado e utilizado no cuidado relacionado à situação crônica de saúde ${ }^{23}$.

Alterações na imagem corporal sinalizam que os profissionais de saúde devem prestar assistência humanizada, personalizada e sistematizada, não se preocupando apenas com a úlcera, mas sim com o indivíduo como um todo, o qual se sente isolado, carente, impotente, e às vezes sem tratamento adequado.

As limitações do estudo aqui tratado incluem o número pequeno de participantes e a ausência de um grupo-controle, sendo esses aspectos uma possível perspectiva para novos estudos.

\section{Conclusão}

Por meio do estudo ora reportado, podemos concluir que as pessoas com úlcera venosa que participaram do estudo apresentaram valores baixos para o escore total médio e escores dos domínios da escala BIS. Tal resultado indica que os indivíduos avaliados têm imagem corporal baixa e seus sentimentos são negativos em relação ao próprio corpo.

Diante das necessidades surgidas nas últimas décadas com o aumento do número de pacientes com insuficiência venosa que podem levar o indivíduo a conviver com a úlcera venosa, torna-se imprescindível redirecionar a formação acadêmica e a qualificação dos profissionais de saúde, valorizando não somente o conteúdo, mas também a prática assistencial prestada a essas pessoas, sendo essencial que esses profissionais tornem-se conscientes da importância de o paciente realizar o autocuidado.

\section{Conflitos de interesses}

Nenhum conflito financeiro, legal ou político envolvendo terceiros (governo, empresas e fundações privadas, etc.) foi declarado para nenhum aspecto do trabalho submetido (incluindo, mas não se limitando a subvenções e financiamentos, participação em conselho consultivo, desenho de estudo, preparação de manuscrito, análise estatística, etc.).

\section{Referências}

1. Cardoso LV, Godoy JMP, Godoy MFG, Czorny RCN. Compression therapy: Unna boot applied to venous injuries: an integrative review of the literature. Rev Esc Enferm. 2018; 52:e03394. doi: 10.1590/S1980-220X2017047503394

2. Salomé GM, Ferreira LM. Impact of non-adherent Ibuprofen foam dressing in the lives of patients with venous ulcers. Rev Col Bras Cir. 2017;44(2):116-24. doi: 10.1590/0100-69912017002002

3. Joaquim FL, Silva RMCRA, Pereira ER, Garcia-Caro MP, CruzQuintana F. Application of Merleau-Pontyan perspective on the physical and psychological implications of venous ulcers. Rev Bras Enferm. 2018;71(5):2469-76. doi: 10.1590/0034-7167-2017-0542 
4. Salomé GM, Ferreira LM. The impact of decongestive physical therapy and elastic bandaging on the control of pain in patients with venous ulcers. Rev Col Bras Cir. 2018;45(2):e1385. doi: $\underline{10.1590 / 0100-6991 e-20181385}$

5. Pereira MTJ, Salomé GM, Openheimer DG, Espósito VHC, Almeida SA, Ferreira LM. Feelings of powerlessness in patients with diabetic foot ulcers. Wounds. 2014;26(6):172-7.

6. Silva MH, Jesus MCP, Merighi MAB, Oliveira DM. The self-care experience of women living with chronic venous leg ulcer. Rev Estima. 2016;14(2):61-7. doi: 10.5327/Z1806-3144201600020003

7. Borges EL, Santos $C M$, Soares MR. Modelo $A B C$ para o manejo da úlcera venosa de perna. Rev Estima. 2017;15(3):182-87. doi: 10.5327/Z1806-3144201700030010

8. Salomé GM, Almeida AS, Ferreira LM. Association of sociodemographic factors with hope for cure, religiosity, and spirituality in patients with venous ulcers. Adv Skin Wound Care. 2015;28(2):76-82.

9. Cifuentes, JEC, Gamboa SG. Factores psicosociales en los pacientes con úlceras venosas y su asociación con la cicatrización. ESTIMA Braz J Enterostomal Ther. 2020; 18:e0720. doi: 10.30886/ estima.v18.845_ESP

10. Faria EC, Loiala T, Salomé GM, Ferreira LM. Unna boot therapy impact on wellbeing, hope and spirituality in venous leg ulcer patients: a prospective clinical trial. Journal Of Wound Care. 2020; 29(4):214-220. doi: 10.12968/jowc.2020.29.4.214

11. Bueno MLGB, Salome GM, Ferreria LM. Effects of Acupuncture on Quality of Life and Pain in Patients with Venous Ulcers. Biomed Sci \& Tech Res. 2018;8(3). doi: 10.26717/BJSTR.2018.08.001641

12. Aguiar ACSA, Sadigursky D, Martins LA, Menezes TMO, Santos ALS, Reis LA. Social repercussions experienced by elderly with venous ulcer. Rev Gaúcha Enferm. 2016;37(3):e55302. doi: 10.1590/1983-1447.2016.03.55302

13. Alves SG, Gardona RGB, Reis BC, Vilela LHR, Salomé GM. Association of sociodemographic effects and injury to feelings of powerlessness and hope in individuals with venous ulcers. Rev Bras Cir Plast. 2013;28(4):672-80.
14. Gouveia VV, Santos CA, Gouveia RSV, Santos WS, Pronk SL. Body investment scale (BIS): Evidences of its factor validity and reliability. Aval Psicol. 2008;7(1):57-66.

15. Silva MH, Jesus MCP, Oliveira DM, Merighi MAB. Unna's boot: experience of care of people with venous ulcers. Rev Bras Enferm. 2017;70(2):366-73. doi: 10.1590/0034-7167-2016-0219

16. Souza AJG, Campos SMDL, Salvetti MG, Queiroz CG, Torres GV, Costa IKF. Autoestima de pessoas com úlcera venosa. Rev Rene. 2017;18(5):569-76. doi: 10.15253/2175-6783.2017000500002

17. Corrêa NFM, Brito MJA, Resende MMC, Duarte MF, Santos FS, Salomé GM et al. Impact of surgical wound dehiscence on health-related quality of life and mental health. J Wound Care. 2016;25(10):561-70. doi: 10.12968/jowc.2016.25.10.561

18. Vilela LHR, Salomé GM, Pereira RC, Ferreira LM. Pain assessment in patients with venous leg ulcer treated by compression therapy with Unna's boot. J Tissue Sci Eng. 2016;7:171. doi: 10.4172/2157-7552.1000171

19. Salomé GM, Almeida SA, Pereira MTJ, Massahud Jr MR, Moreira CNO, Brito MJA et al. The impact of venous leg ulcers on body image and self-esteem. Adv Skin Wound Care. 2016;29(7):316-21. doi: 10.1097/01.ASW.0000484243.32091.0c

20. Silva MC, Salomé GM, Miguel P, Bernardino C, Eufrásio C, Ferreira LM. Evaluation of feelings helplessness and body image in patients with burns. J Nurs UFPE. 2016;10(6):2134-40. doi: 10.5205/1981-8963-V10I6A11227P2134-2140-2016

21. Salomé GM, Almeida SA, Mendes B, Carvalho MRF, Bueno JC, Massahud Jr MR et al. Association of sociodemographic factors with spirituality and hope in patients with diabetic foot ulcers. Adv Skin Wound Care. 2017;30(1):34-39. doi: 10.1097/01. ASW.0000508446.58173.29

22. Moreira CNO, Marques CB, Salomé GM, Cunha DR, Pinheiro FAM. Health locus of control, spirituality and hope for healing in individuals with intestinal stoma. J Coloproctol. 2016;36(4):208-15. doi: $10.1016 / j . j$ col.2016.04.013

23. Dias TYAF, Costa IK, Salvetti MG, Mendes CKTT, Torres GV. Influences of health care services and clinical characteristics. Rev Paul Enferm. 2013;26(10):529-34. doi: 10.1590/S010321002013000600004 